\title{
Kinetics of Change in Droplet Size during Nanoemulsion Formation
}

\author{
Ankur Gupta, Vivek Narsimhan, T. Alan Hatton, and Patrick S. Doyle* \\ Massachusetts Institute of Technology, Cambridge, MA 02139 \\ E-mail: pdoyle@mit.edu
}

\section{Choosing Initial Droplet Size Distribution}

The homogenizer and ultrasonicator feed was analyzed under a microscope and droplet size distribution was arrived at using image analysis. The assumed distribution is close the experiments (Fig.S1 (a)). Also, the final droplet size distribution is independent to the choice of initial droplet size distribution (Fig.S1 (b)). Please note that the final distribution from all the three cases overlap with each other and thus two of them are not visible in the plot.

\section{Droplet size data for different $\varepsilon$ input}

The variation of $d_{\text {avg }}$ with pressure drop $(\Delta P)$ in the homogenizer and amplitude $(A)$ in the ultrasonicator are provided in Fig.S2 (silicone oil with viscosity $4 \mathrm{mPa}$.s was used for the experiments). For the homogenizer, $d_{a v g}$ decreases initially with increasing number of passes $N$ and then becomes roughly constant. The steady state droplet size decreases with increasing $\Delta P$ as expected since $\Delta P \propto \varepsilon$ (Fig.S2 (a)). For the ultrasonicator, $d_{\text {avg }}$ decreases

*To whom correspondence should be addressed 
initially with increasing sonication time $t_{u s}$ and then becomes roughly constant. However, the steady state is roughly constant with amplitude $(A)$ as shown in Fig.S2 (b). Lastly, the decay rate of $d_{\text {avg }}$ does not depend on either $\Delta P$ or $A$. Also, polydispersity stays roughly constant with $N$ and $t_{u s}$.

\section{Droplet size data plotted with energy input}

As mentioned in the Introduction, some reports in the literature incorrectly assume that energy input is the only parameter that controls the evolution of average droplet size. Here

we plot our $d_{\text {avg }}$ data from homogenizer with energy input density where we observe a large spread in the experimental data due to the effect of $\mu_{d}$ (Fig.S3).

\section{Parity plot for ultrasonicator data}

A plot similar to Fig.7 can also be obtained for ultrasonication data (Fig.S4) using the following equation:

$$
d_{\text {avg, fit }}(N)=C_{1} \frac{\sigma^{5 / 6} \mu_{d}^{1 / 3}}{\left(\rho_{d} \sigma\right)^{1 / 6}\left(\mu_{c} \rho_{c} \varepsilon\right)^{5 / 12}}+C_{2} \exp \left(-\frac{t_{u s}}{t_{b}}\right)
$$

Similar to homogenizer, the disagreement is higher for low Oh. The values of parameters $C_{1}, C_{2}$ and $t_{b}$ are $0.10,411 \mathrm{~nm}$ and 2.9 min respectively. 

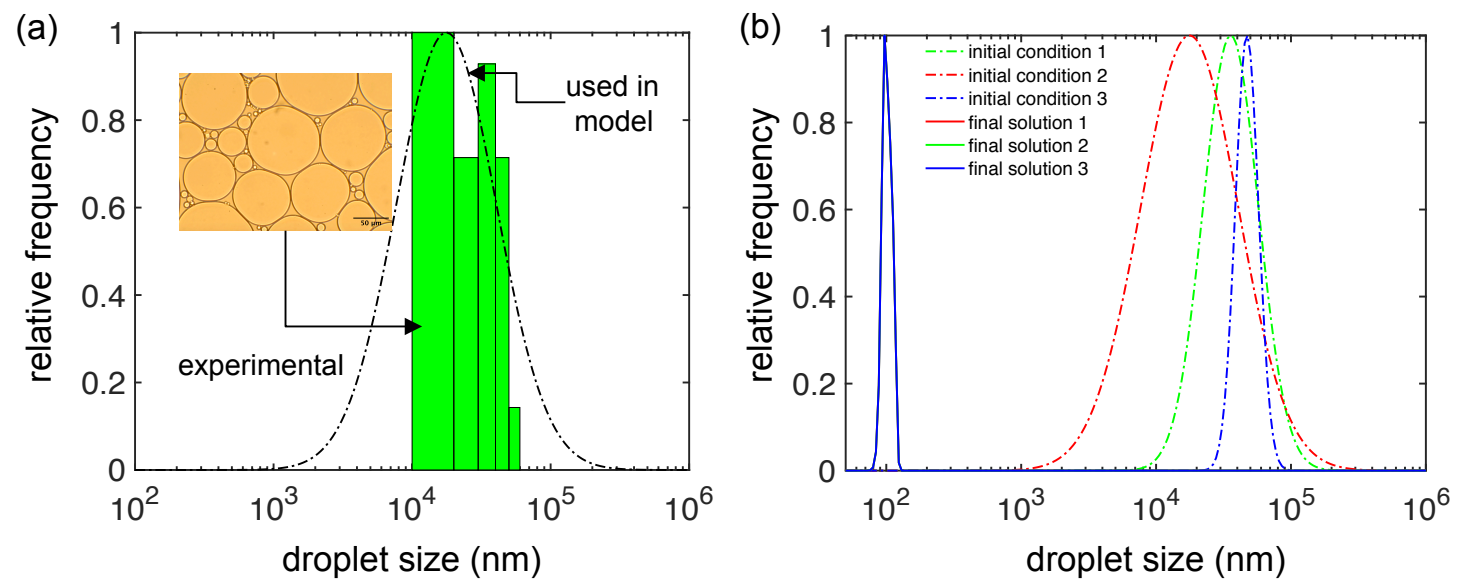

Figure S1: (a) Comparison of assumed and experimental initial droplet size distribution (b) Plot demonstrating that final droplet size is insensitive to the choice of initial droplet size distribution. The distributions are plotted by dividing $n_{i}$ (Equation (16)) by $n_{i, \max }$ so that the maximum value is always unity. Please note that the final distribution from all the three cases overlap with each other and thus two of them are not visible in the plot. 
(a) homogenizer

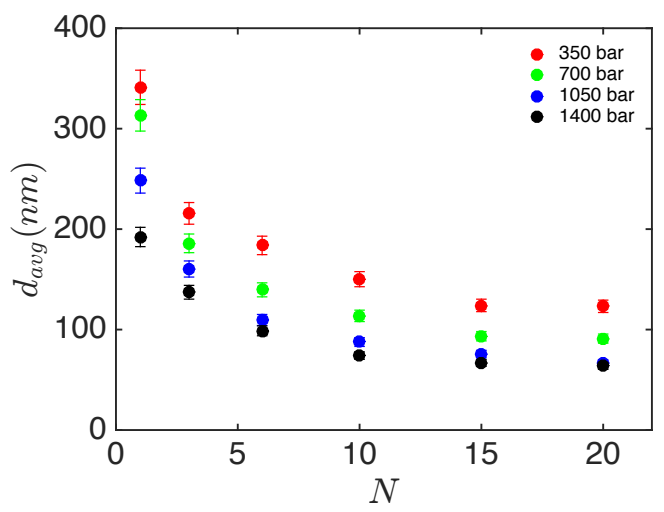

(b) ultrasonicator

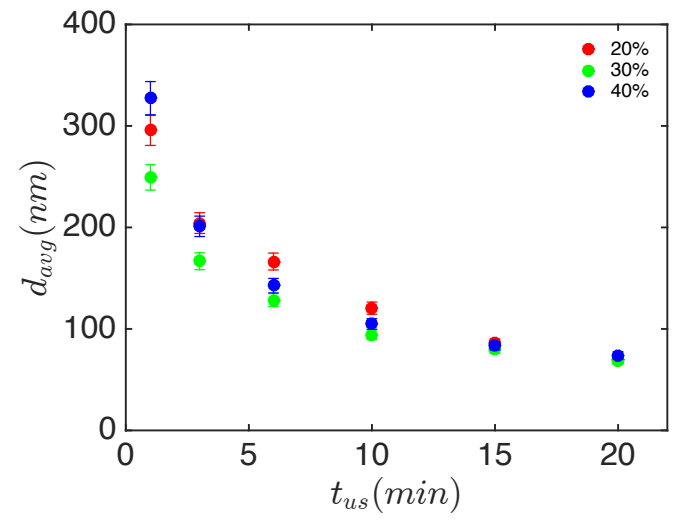

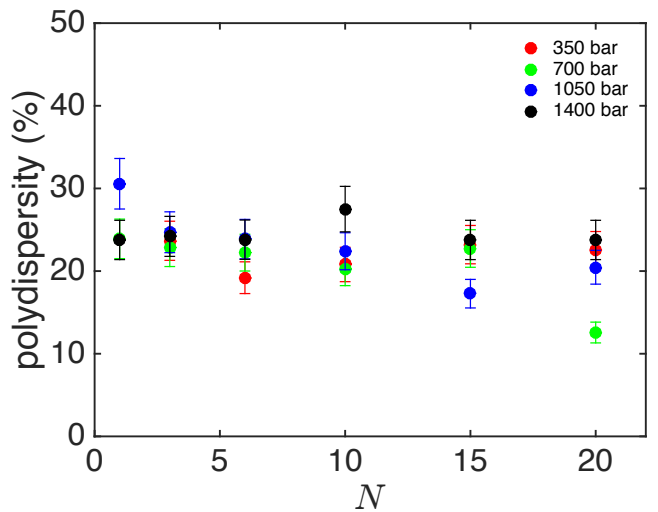

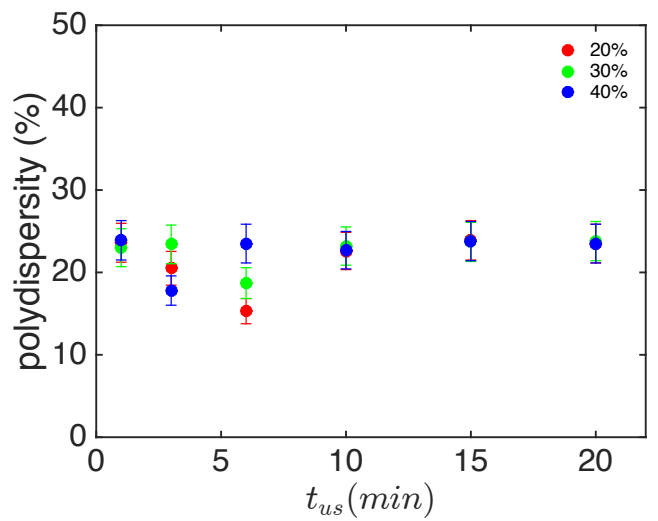

Figure S2: (a) $d_{\text {avg }}$ and polydispersity profiles for different $\Delta P$ in a homogenizer. The final droplet size deceases with increase in $\Delta P$ since $\varepsilon \propto \Delta P$. The rate of droplet size decay appears to be independent of $\Delta P$. Polydispersity does not show any significant trend with $N$. (b) $d_{\text {avg }}$ and polydispersity profiles for different $A$ in a homogenizer. The final droplet size remains constant with change in $A$ since $\varepsilon$ is a weak function of $A$. The rate of droplet size decay appears to be independent of $A$. Polydispersity does not show any significant trend with $A$. 


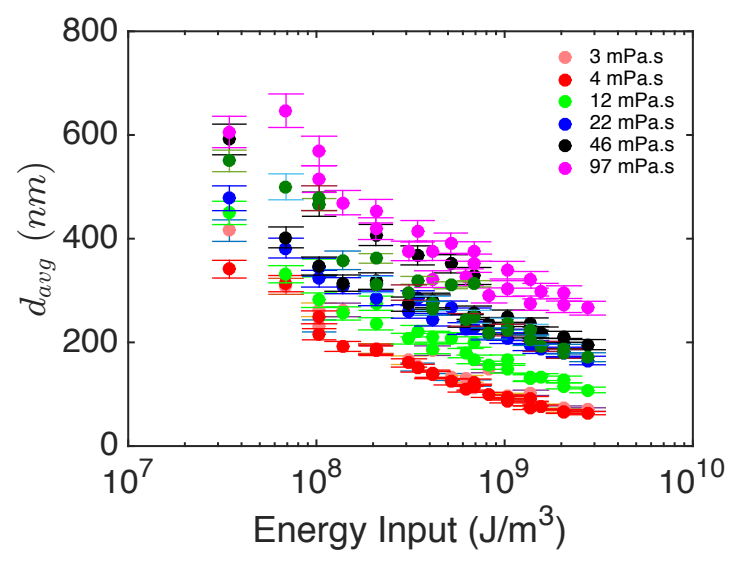

Figure S3: Comparison of $d_{\text {avg }}$ from homogenizer with energy input density for different $\mu_{d}$.

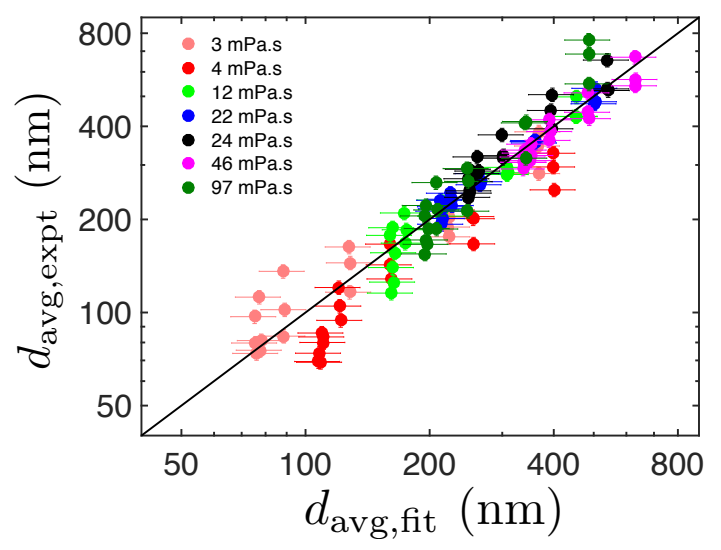

Figure S4: A parity plot for kinetic droplet size data prepared with different $\mu_{d}$, $\varepsilon$, and $N$. We obtain very good agreement between $d_{\mathrm{avg} \text {, fit }}$ and $d_{\mathrm{avg} \text {, expt }}$ using $C_{1}=0.10, C_{2}=411 \mathrm{~nm}$, $N_{b}=2.9$ min. 